\title{
Matters of significance
}

\author{
Sound experimental design and analysis require improved statistical training.
}

Concerns about data quality and reproducibility in biomedical research have been rising. This May, Nature Publishing Group put in place new reporting standards for the research we publish. At the core of these standards is a document that asks authors to disclose technical and statistical information about their study. But because these reporting requirements come after research is completed and the manuscript is written and submitted for publication, they do not actually affect experimental design but rather serve to better expose the existing level of rigor to reviewers and readers.

In an effort to give these topics the attention they deserve and help researchers at the stage of experimental planning and design, we debut a new column: Points of Significance. The column will present important concepts and practical advice about statistics and experimental design in an easily digestible format.

As biological researchers apply increasingly refined techniques such as targeted genome engineering that are likely to yield smaller but more biologically meaningful effects, study design and analysis decisions are more important than ever. Experimentalists are also examining systems at a depth that is orders of magnitude greater than that of just five years ago. Analyzing such data will require pushing the envelope in experimental design standards and analysis.

Fortunately, the necessary understanding of basic concepts of variability, effect size and experimental design essential for guiding good experimental practice can be gained with minimal mathematical sophistication. For much bench research, these fundamental principles inform the design of valid replicable experiments that can be analyzed using standard techniques. But scientists should also know enough to realize when their level of training is insufficient and it is time to talk to a statistician. For large studies, a discussion with a statistician at the study design stage-as is commonly done for clinical studies - can save resources and money and prevent angst.

A considerable amount of basic research flies by the seat of its pants, performed while techniques are still being developed and while it isn't yet known whether usable data will be forthcoming. In these cases, in which statistical consultation may be difficult or inefficient, a basic understanding of statistical concepts can help guide the experimental process and allow the researcher to avoid unproductive or misleading paths of investigation.

Because our intuition about probability can be misguided (p. 809), some form of training is essential for developing a good grasp of fundamental statistical concepts and practice.
But despite perennial grumbling about inadequate statistical competence among biological researchers, statistics training is often not part of the core course requirements in biological graduate programs.

A challenge to providing universal training is the difficulty in offering a single course that covers the technical requirements of different fields of biology and provides engaging teaching examples that all students can relate to. Statistics training is thus often relegated to required discipline-specific methodology courses, or students must take available statistics electives. As a result, a substantial number of practicing researchers in biology end up with no formal statistics training.

Basic training in experimental design and statistics should be required in all graduate programs that frequently lead to careers in biological research. Scientific ethics courses are now a core part of many graduate biomedical programs. These could integrate instruction about experimental design and basic statistics to complement ethical considerations in making important decisions at each stage of a research project regarding trade-offs among sample size, methodologies and available time and resources. Because those decisions are intimately connected to the reliability of the results, they possess an inherent ethical element that may not be appreciated by researchers anxious to get results. Tying experimental design and statistics to discussion of scientific ethics could lead to greater appreciation of their importance. However, the move in the United States to external online courses for ethics training makes this fusion difficult; thus, a better solution is a dedicated experimental design course that presents design, statistics and ethics in a holistic manner.

But what should practicing researchers with no formal training in statistics do? There is no shortage of statistics books targeted at biologists. A search of Amazon. com using "statistics for biology" gives no less than 3,000 results, with the top result appropriately titled Statistics for Terrified Biologists. Online courses are another option for obtaining the necessary minimum training, but it is difficult for someone immersed in research to make the time commitment either option requires.

We hope that by following in the footsteps of the successful Points of View column on visualization-now organized for browsing on Methagora-that the new Points of Significance column can fill a need and encourage both busy researchers and students to think more about statistics and gain a deeper appreciation of how they can improve the experimental rigor of their work. 